\title{
Local Size Segregation in Polydisperse Hard Sphere Fluids
}

\author{
I. Pagonabarraga, M.E. Cates, and G. J. Ackland \\ Department of Physics and Astronomy, JCMB The King's Buildings, Mayfield Road, EH9 3JZ Edinburgh, United Kingdom
}

(Received 3 August 1999)

\begin{abstract}
The structure of polydisperse hard sphere fluids, in the presence of a wall, is studied by the Rosenfeld density functional theory. Within this approach, the local excess free energy depends on only four combinations of the full set of density fields. The case of continuous polydispersity thereby becomes tractable. We predict, generically, an oscillatory size segregation close to the wall, and connect this, by a perturbation theory for narrow distributions, with the reversible work for changing the size of one particle in a monodisperse reference fluid.
\end{abstract}

PACS numbers: 61.20.Gy, 05.20.Jj, 64.75.+g

Understanding the behavior of polydisperse systems is relevant to many materials of practical interest. In particular, colloidal and/or polymeric fluids generally contain particles which have, in effect, a continuous distribution of sizes (and/or other parameters such as charge and chemical composition). This affects their performance in applications ranging from foodstuffs to polymer processing [1]. More fundamentally, colloidal systems also provide the closest experimental approach to the "theorists ideal fluid," namely, that of perfect hard spheres [2]. The fact that all colloids are in practice polydisperse (at least slightly) must then be taken into account in comparing theory with experiment. Only recently has experimental work started to clarify in a systematic way the generic consequences of polydispersity, such as the partitioning of sizes between coexisting phases [3].

Despite the continuous interest polydisperse fluids have raised, their theoretical understanding remains far from complete, especially for inhomogeneous cases. More is known about partial structure factors in single-phase fluids $[4,5]$ and liquid-liquid phase equilibrium [6,7] than about crystalline phases [8] or interfacial properties, for example. And, where such inhomogeneous situations have been studied [9] it has often proved necessary to assume that only the mean density, and not the size distribution, can vary in space [10]. This ignores size segregation effects, which (globally) influence the phase diagram $[3,7]$. A similar tendency to local segregation is implicit in treatments of binary and ternary hard sphere mixtures [11-13] and in polydisperse equilibrium structure factors in the homogeneous state [4].

In what follows, we treat continuous polydispersity within a density functional theory (DFT) that properly allows for local size segregation. Our work is based on a choice of density functional (that of Rosenfeld [14]) that has previously been used to study finite mixtures of hard spheres. By exploiting the fact that its excess free energy density depends on only a small number of linear combinations of the particle densities (four "moment densities"), we are able to address the case of continuous polydispersity, where the underlying densities are infinite in number. This allows us to study, e.g., the effects of varying the shape of a smooth size distribution. Moreover, by a perturbative analysis of the same functional, we can distinguish certain generic features that do not depend on the shape of the parent, if it remains narrow. These two aspects of our work build on recent nonperturbative [15] and perturbative $[3,16]$ progress in understanding polydisperse phase equilibria.

The state of a polydisperse fluid is specified by the local number density of each species, $\rho(\sigma, \mathbf{r})$, with $\sigma$ the particle diameter (let us say). The spatial average of this quantity must recover the (known) global size distribution, $\rho(\sigma)$, which we call the "parent." According to DFT, the grand potential is some (unknown) functional of $\rho(\sigma, \mathbf{r})$. Given an approximation to this functional, the key problem of polydispersity is the need to find, by its minimization, an infinite set of densities at each point in space. A naive discretization into, let us say, $N=50$ species does not make this much easier: the minimization problem remains of very high dimension (we have $N$ functions of $d$ spatial coordinates, with $d$ the effective space dimension; $d=1$ for a fluid near a flat wall).

The problem of free energy minimization in a very large space arises already in the calculation of phase diagrams, where it has been found that, for many purposes, the problem can be exactly projected onto a subspace involving a few linear combinations (or "moments") of $\rho(\sigma)$ [15]. The approach requires that the excess free energy can be expressed in terms of the chosen moments; this is true for many approximate theories including polymer mean-field theories and liquid state models such as the Percus-Yevick equation of state (PY) [17,18].

We now observe that essentially the same simplification is possible with certain (approximate, but wellestablished) density functionals for inhomogeneous liquids [17]. We choose for definiteness that of Rosenfeld [14] for hard spheres, as subsequently recast by Kierlik and Rosinberg [19], whose nonideal part is a functional of four moment densities defined, from the underlying density profile $\rho(\sigma, \mathbf{r})$, as follows (with $\alpha=0,1,2,3$ ):

$$
m_{\alpha}(\mathbf{r})=\int d \sigma d \mathbf{r}^{\prime} \rho\left(\sigma, \mathbf{r}^{\prime}\right) \omega_{\alpha}\left(\sigma,\left|\mathbf{r}^{\prime}-\mathbf{r}\right|\right)
$$


Here $\omega_{\alpha}\left(\sigma,\left|\mathbf{r}^{\prime}-\mathbf{r}\right|\right)$ are four weight functions, selected $[14,19]$ such that PY is recovered for a homogeneous mixture. Note that only four moment densities are needed, irrespective of the number of components of the mixture; this follows from PY itself, whose nonideal part $\mathcal{F}^{\mathrm{ex}}\left(m_{\alpha}\right)$ involves only the four moments $m_{\alpha}=\int d \sigma \rho(\sigma) \sigma^{\alpha}$. However, the weight functions $\omega_{\alpha}$ are nonlocal: our moment densities $m_{\alpha}(\mathbf{r})$, though intimately related to the four PY moments, are not merely local values of them.

Within this description, the grand potential becomes

$$
\begin{aligned}
\Omega= & \int d \mathbf{r} d \sigma\left\{\rho(\sigma, \mathbf{r})\left\{\ln \left[\Lambda^{3}(\sigma) \rho(\sigma, \mathbf{r})\right]-1\right\}\right. \\
& +[V(\sigma, \mathbf{r})-\mu(\sigma)] \rho(\sigma, \mathbf{r})\} \\
+\int d \mathbf{r} \mathcal{F}^{\operatorname{ex}}\left(m_{\alpha}(\mathbf{r})\right) &
\end{aligned}
$$

where $\mu(\sigma)$ is the chemical potential of species $\sigma$ in the bulk, $\Lambda(\sigma)$ is a thermal wavelength [6], $V(\sigma, \mathbf{r})$ is the external potential acting on species $\sigma$, and $\mathcal{F}^{\text {ex }}\left(m_{\alpha}\right)=$ $-m_{0} \ln \left(1-m_{3}\right)+m_{1} m_{2} /\left(1-m_{3}\right)+m_{2}^{3} /\left[24 \pi\left(1-m_{3}\right)^{2}\right]$ gives the usual PY result [17]. Minimization of Eq. (2) leads to Eq. (1) with

$$
\rho(\sigma, \mathbf{r})=R(\sigma) \exp \{-\beta V(\sigma, \mathbf{r})+\tilde{c}(\sigma, \mathbf{r})-\tilde{c}(\sigma, \infty)\},
$$

$$
\tilde{c}(\sigma, \mathbf{r})=-\sum_{\beta} \int d \mathbf{r}^{\prime} \frac{\partial \mathcal{F}^{\mathrm{ex}}}{\partial m_{\beta}}\left(\mathbf{r}^{\prime}\right) \omega_{\beta}\left(\sigma,\left|\mathbf{r}^{\prime}-\mathbf{r}\right|\right) .
$$

Here $\tilde{c}(\sigma, \infty)$ is the value of the excess chemical potential, and $R(\sigma)$ is the density distribution, in the bulk.

For a localized potential $V$ (such as a hard wall), and for any other case where the densities differ from their bulk values only in a finite neighborhood, $R(\sigma)$ coincides with the parent $\rho(\sigma)$ in the thermodynamic limit [20], and is known in advance. Equations (1), (3), and (4) are then closed in the low-dimensional function space spanned by $\left\{m_{\alpha}(\mathbf{r})\right\}$. Their numerical evaluation delivers [via Eq. (3)] the full density profile, but nonetheless proceeds as if there were only four species present. Note that the four momentdensity profiles depend [through Eqs. (1) and (3)] on $R(\sigma)$, so that the final results, even for the $m_{\alpha}(\mathbf{r})$ themselves, still depend on all moments of the parent distribution.

Of course, not all density functionals in common use are of the required "moment density" form [17]. But Eq. (2) performs as well as most other functionals proposed in the literature [13]; for example, depletion forces in binary fluids are well recovered by it [21]. For numerical use below we retain the original weight functions [14], although some recent modifications are known to give a better description of solid phases [22]; these modifications would not alter the conceptual structure of our analysis.

We now focus on the effect of polydispersity in a fluid of hard spheres near a flat hard wall. We fix the parent size distribution $R(\sigma)=\rho(\sigma, \infty)$, and thereby the chemical potentials of all species in the bulk, and analyze the structure as a function of distance $z$ from the wall. We have considered fluids with a Gaussian, an exponential, and a uniform (top hat) distribution of sizes; each is characterized by its mean (which we set as $\bar{\sigma}=1$ without loss of generality) and its standard deviation (which is then called the polydispersity, $p$, and is expressed in percent). We disregard any transverse ordering, which is reasonable for a fluid, except possibly close to the fluid/solid transition.

For all the parents considered, we find significant local size segregation, with a strong, local cross correlation between particles of different sizes. This effect can be observed in Fig. 1, where we display the local relative concentration of various species, $\phi(\sigma, z)=$ $\rho(\sigma, z) / \int d \sigma \rho(\sigma, z)$, for the case of a top-hat parent with $p=11.5 \%$. Clearly visible are strong, anticorrelated oscillations in the relative amounts of large and small particles, whereas the relative concentration of particles close to the mean size $(\sigma=1)$ is much more nearly constant. Our definition of $\phi(\sigma)$ factors out the primary oscillations in the mean density (depicted in Fig. 2), but we find that the size distribution (Fig. 1 insets) oscillates roughly in quadrature to the mean density. In this manner, without greatly altering the overall density profile, smaller particles are accommodated on the inner (near-wall) side of each successive density peak and larger particles on the outer side. Thus the density of each species can, within the first few layers at least, oscillate with a spatial period close to its own diameter. (The same could not continue indefinitely: the nature of any size segregation in ordered phases thus remains an important, open issue. Moreover, the asymptotic decay of the density profile for

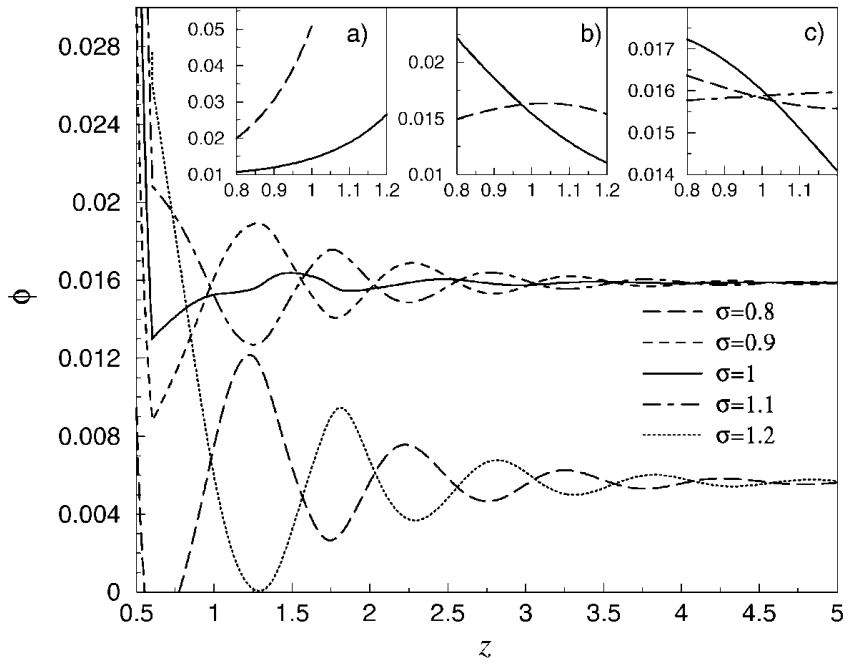

FIG. 1. Profiles for the local concentration of several species for a flat parent with $p=11.5 \%$, at mean volume fraction $\eta=0.4$. The mean diameter is taken as the length unit. The curves for $\sigma=0.8$ and $\sigma=1.2$ have been vertically displaced to aid comparison. Insets: Size distribution at different $z$. (a) Dashed line, $z=0.5$; solid line, $z=0.8$. (b) Solid line, $z=1.2$; dashed line, $z=1.6$. (c) Solid line, $z=2.4$; dashed line, $z=3.2$; dotted-dashed line, $z=4.7$. 


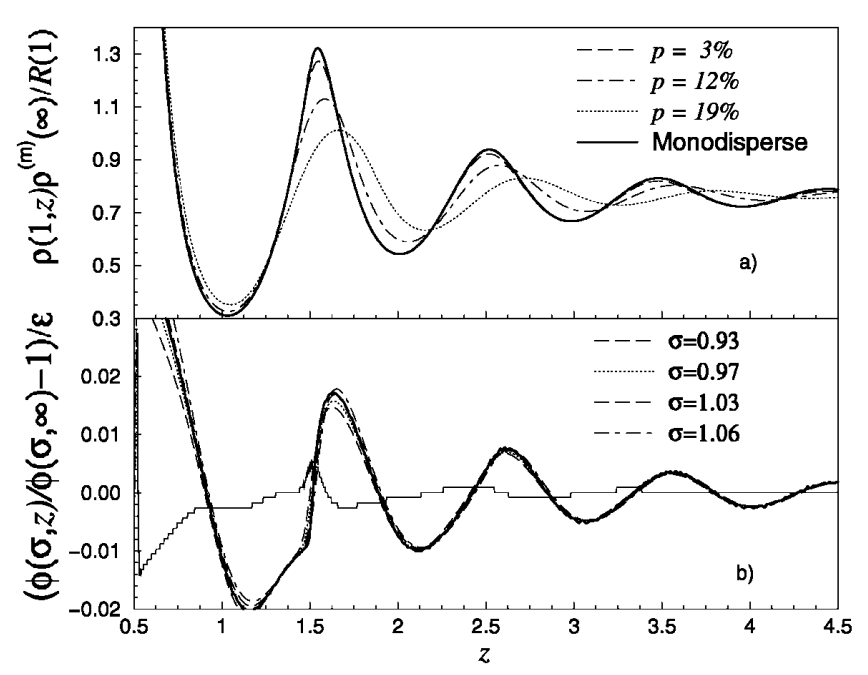

FIG. 2. (a) Density profiles for the mean species with a flat parent, setting $\bar{\sigma}=1$, compared with the profile of a monodisperse fluid at the same mean volume fraction, $\eta=0.4$. (b) Reduced local concentration profiles, for a Gaussian parent $(p=2.4 \%)$ at $\eta=0.45$, compared with $\tilde{c}^{\prime}(z)-\tilde{c}^{\prime}(\infty)$ from the monodisperse fluid at the same $\eta$, thick continuous line. The thin continuous line refers to the local concentration of the mean species. This curve is not reduced but has been shifted vertically so that it tends to zero at large $z$.

any mixture is given by a single wavelength [23].) As the polydispersity of the parent increases, the trend we have outlined persists, although it blurs, and the uniform relative concentration of the mean species is lost. The behavior is generic for all the different size distributions tried and can also be seen in ternary (or even binary) mixtures [13].

We can gain further insight into this behavior by a perturbative analysis for a narrow parent, analogous to that used previously for phase coexistence [16]. We express the particle diameters as $\sigma=\bar{\sigma}(1+\epsilon)$; we then expand in small $\epsilon$ around a monodisperse density profile for that species. The perturbative result is (to order $\epsilon$ ) [13]

$$
\begin{aligned}
\rho(\sigma, z)= & \frac{R(\sigma)}{\rho^{(m)}(\infty)} \rho^{(m)}(z) e^{-\beta[V(\sigma, z)-V(\bar{\sigma}, z)]} \\
& \times\left\{1+\epsilon\left[\tilde{c}^{\prime}(z)-\tilde{c}^{\prime}(\infty)\right]\right\}
\end{aligned}
$$

Here $\rho^{(m)}(z)$ is the density profile for the monodisperse system, and $\tilde{c}^{\prime}(z)$ is the reversible work coefficient for slightly changing the size of one particle, $z$ from the wall, within an otherwise monodisperse system. This obeys

$$
\tilde{c}^{\prime}(\mathbf{r})=\left.\frac{\partial}{\partial \epsilon} \tilde{c}(\sigma, \mathbf{r})\right|_{\epsilon=0, p \rightarrow 0},
$$

which can easily be computed from Eq. (4). We have assumed that $\epsilon \ll 1$ for all members of the parent, but not that $\epsilon \ll p$; this is why there is no expansion made of the factor $R(\sigma)$ in Eq. (5). Likewise for a hard wall it is inappropriate to expand $V(\sigma, z)$.
These perturbative calculations predict that the profile of the "mean species" is, to order $\epsilon$, identical to a monodisperse fluid at the same overall density. This agrees with Fig. 2a, where we compare the mean-species profiles for flat parents of varying polydispersity. Curves for other parents (not shown), but with matched polydispersity, are barely distinguishable on such a plot, even when this is over $10 \%$. More generally, Eq. (5) shows that all the order $\epsilon$ deviations from monodisperse behavior, for parents of different shapes, should fall on a common curve if the densities $\rho(\sigma, z)$ are first normalized by $R(\sigma)$. We have verified this by comparing $\rho(\sigma, z) / R(\sigma)$ for small fixed $\epsilon$, among parents of different shapes [13].

An illuminating application of the perturbation theory is to the local concentration profiles shown in Fig. 1. According to Eq. (5), in any region where the external potential does not depend on the radius of the particles,

$$
\phi(\sigma, z)=\phi(\sigma, \infty)\left\{1+\epsilon\left[\tilde{c}^{\prime}(z)-\tilde{c}^{\prime}(\infty)\right]\right\},
$$

which shows, to order $\epsilon$, that the local concentration of the mean species is strictly constant, and that by an appropriate scaling, the concentration profiles for different particle sizes collapse onto a single curve. These predictions are confirmed from the full (nonperturbative) solution for a narrow Gaussian parent in Fig. 2b. The size oscillations are, as predicted, directly linked to the reversible work term, $\tilde{c}^{\prime}(z)$, for changing the size of one particle. Although the data collapse is mostly excellent, the constancy of the mean species concentration is imperfect close to the wall even for narrow (2.4\%) polydispersity. This may be because the perturbative expansion itself fails very close to a hard wall, where the potential forces the concentration of certain species to be zero. (This is visible in the first inset in Fig. 1.) The same problem should not arise for a smoothly varying potential.

We now turn to thermodynamic properties of our system. We have integrated the density and the energy profiles to find the adsorption $\Gamma$ and the surface tension, $\gamma$ [17]. In Fig. 3 we show the $\Gamma$ as a function of the volume fraction, for different parents and polydispersities. It appears that, until the parent becomes relatively wide, the values of the measured adsorptions do not differ significantly from the monodisperse case. Indeed, our perturbative calculations show the deviation, and that of the surface tension [13], to be of order $p^{2}$. But in fact the deviations are only small if, as shown, one uses well-chosen moments to scale the plot (effectively, $\Gamma m_{2} / m_{0}$ vs $m_{3}$ ). These scalings are suggested by, e.g., scaled particle theories for the adsorption [24]; with different choices, there are deviations of up to $30 \%$ in the same data. The same generic features are observed for the surface tension, displayed in the inset in Fig. 3. For the exponential parent, as the polydispersity increases, the surface tension becomes more negative.

In this Letter we have considered the effect of polydispersity on the structure of an inhomogeneous hard sphere 


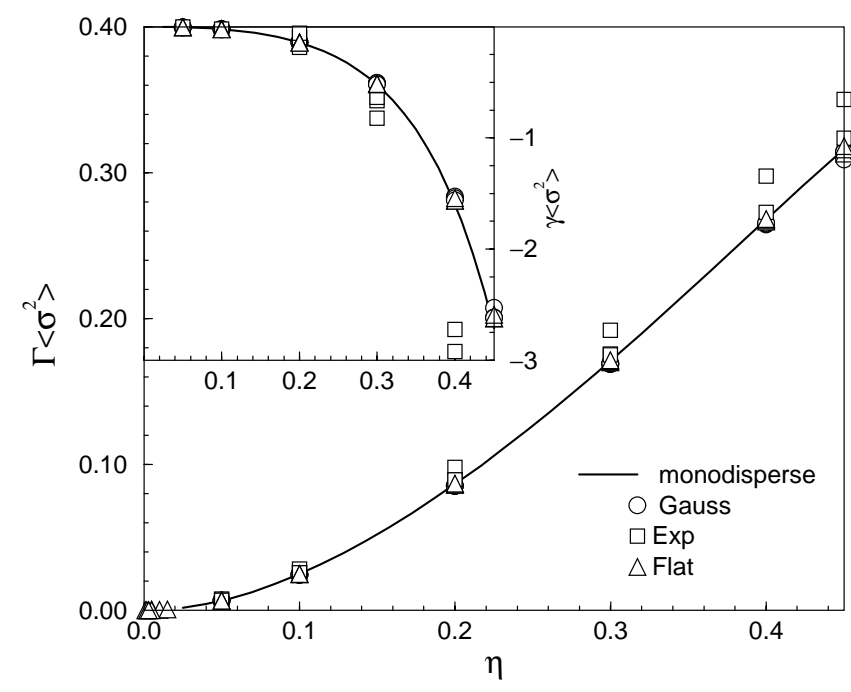

FIG. 3. Adsorption for different parents. Gaussian: $2.4 \%, 7 \%$, 12\%; exponential: $9 \%, 23 \%$ (cutoff at a minimum $\sigma$ of 0.91 and 0.77 , respectively), flat: $4 \%, 12 \%, 19 \%$. Inset: surface tension for the same parents.

fluid. We have shown that the use of moment densities makes DFT a practical tool to study inhomogeneous fluids of continuous polydispersity, in which local size segregation can play a major role. This was illustrated by studying a polydisperse fluid near a wall, which clearly shows such effects (see Fig. 1). A perturbative analysis confirmed the generic features of such segregation. We can also obtain a general relation that connects the ratios of differences in the local moments [evaluated from $\rho(\sigma, \mathbf{r})$ at two different points] to ratios of higher moments evaluated for the parent [13]. Such findings generalize similar $\mathcal{O}(\epsilon)$ results for phase partitioning [3,16]; for these purposes, it is as if, in DFT, each point in space counts as a different "phase."

We note finally that some of our perturbative results (including those just described) are surprisingly general. For example, Eq. (5), which connects the species densities to the local reversible work of enlarging a particle in the monodisperse limit, makes no assumption about the choice of density functional. Hence it holds for the true functional, whatever it may be [25]. Thus the choice of physical system (hard spheres), and of approximate functional [Eq. (2)] enters only through the particular form of $\tilde{c}^{\prime}(\mathbf{r})$ [Eq. (6)]. Equation (5) is thus an exact result for any slightly polydisperse isotropic fluid; moreover, the polydisperse feature $(\sigma)$ need not even be size, but could be charge, or any other scalar quantity.

We are grateful to P. Bartlett, R. M. L. Evans, P. Sollich, and P. B. Warren for their useful comments. The work was funded by EPSRC GR M/29696.
[1] R. G. Larson, The Structure and Rheology of Complex Fluids (Oxford University Press, New York, 1999).

[2] P. N. Pusey, in Liquids, Freezing and the Glass Transition, edited by J.-P. Hansen, D. Levesque, and J. Zinn-Justin (Elsevier, Amsterdam, 1991).

[3] R. M. L. Evans, D. J. Fairhurst, and W.C. K. Poon, Phys. Rev. Lett. 81, 1326 (1998).

[4] F. Lado, Phys. Rev. E 54, 4411 (1996); S. Leroch, G. Kahl, and F. Lado, Phys. Rev. E 59, 6937 (1999).

[5] B. D'Aguanno and R. Klein, Phys. Rev. A 46, 7652 (1992).

[6] J. J. Salacuse and G. Stell, J. Chem. Phys. 77, 3714 (1982).

[7] J. A. Gualtieri, J. M. Kincaid, and G. Morrison, J. Chem. Phys. 77, 521 (1982).

[8] P. Bartlett, J. Chem. Phys. 109, 10970 (1998); R. P. Sear, Europhys. Lett. 44, 531 (1998).

[9] J. L. Barrat and J.P. Hansen, J. Phys. (Paris) 47, 1547 (1986); R. McRae and A. D. J. Haymet, J. Chem. Phys. 88, 1114 (1988).

[10] Alternatively one works in a grand ensemble and assumes a specific dependence of chemical potential on particle size, but this should in reality be varied to allow an arbitrary (but fixed) parent distribution. See D. A. Kofke and E. D. Glandt, J. Chem. Phys. 90, 439 (1988).

[11] M. Dijkstra, R. van Roij, and R. Evans, Phys. Rev. E 59, 5744 (1999).

[12] A. R. Denton and N.W. Aschcroft, Phys. Rev. A 44, 8242 (1991).

[13] I. Pagonabarraga, M.E. Cates, and G. J. Ackland (to be published).

[14] Y. Rosenfeld, Phys. Rev. Lett. 63, 980 (1989).

[15] P. Sollich and M. Cates, Phys. Rev. Lett. 80, 1365 (1998); P. B. Warren, Phys. Rev. Lett. 80, 1369 (1998).

[16] R. M. L. Evans, Phys. Rev. E 59, 3192 (1999).

[17] R. Evans, in Fundamentals of Inhomogeneous Fluids, edited by D. Henderson (Dekker, New York, 1992), p. 85.

[18] P. B. Warren, Europhys. Lett. 46, 295 (1999).

[19] E. Kierlik and M.L. Rosinberg, Phys. Rev. A 42, 3382 (1990).

[20] The projection method of [15] likewise becomes exact (for systems whose excess free energy has the required form) whenever only a small subvolume deviates (perhaps strongly) from the parent.

[21] B. Götzlemann, R. Roth, S. Dietrich, M. Dijkstra, and R. Evans, Europhys. Lett. 47, 398 (1999).

[22] Y. Rosenfeld, M. Schmidt, H. Löwen, and P. Tarazona, Phys. Rev. E 55, 4245 (1997).

[23] R. Evans, R. J. F. Leote de Carvalho, J. R. Henderson, and D. C. Hoyle, J. Chem. Phys. 100, 591 (1994).

[24] J. R. Henderson and F. van Swol, Mol. Phys. 51, 991 (1984); J. P. Noworyta, D. Henderson, S. Sokolowski, and K.-Y. Chan, Mol. Phys. 95, 415 (1998).

[25] This assumes the relevant derivatives of the exact density functional are finite, even for hard spheres. 